\title{
HLA-Matched and HLA-Haploidentical Hematopoietic Stem Cell Transplantation for Acute Myelogenous Leukemia with $\mathrm{t} \quad(16 ; 21)(\mathbf{p 1 1 . 2 ; q 2 2})$
}

\author{
Yumiko Inui ${ }^{1,2}$, Keiji Matsui ${ }^{3}$, Hisayuki Matsumoto ${ }^{3}$, Taku Nose ${ }^{2}$, Rio Shiraki ${ }^{4}$, Yuji Nakamachi ${ }^{5}$, Katsuya Yamamoto $^{2}$, \\ Atsuo Okamura ${ }^{1,2}$ \\ ${ }^{1}$ Department of Medical Oncology/Hematology, Kakogawa Central City Hospital, Hyogo, Japan \\ ${ }^{2}$ Division of Medical Oncology/Hematology, Department of Medicine, Kobe University Graduate School of Medicine, \\ Kobe, Japan \\ ${ }^{3}$ Department of Clinical Laboratory, Kobe University Hospital, Kobe, Japan \\ ${ }^{4}$ Department of Internal Medicine, Kakogawa Central City Hospital, Hyogo, Japan \\ ${ }^{5}$ BioResource Center, Kobe University Hospital, Kobe, Japan
}

Acute myelogenous leukemia (AML) with $t(16 ; 21)(p 11 ; q 22)$ is a rare leukemia subtype with a relatively high incidence in young individuals and a poor prognosis. This chromosomal rearrangement results in FUS-ERG fusion transcripts. We describe a patient with $A M L$ with $t(16 ; 21)(p 11 ; q 22)$ who underwent successful monitoring for FUS-ERG fusion transcripts by real-time quantitative polymerase chain reaction (RT-qPCR). We designed DNA primers based on the leukemic cells of this patient and monitored minimal residual disease by RT-qPCR. Standard multiagent chemotherapy for AML could not reduce FUS-ERG expression level to $10^{3}$, whereas hematopoietic stem cell transplantation (HSCT) knocked down the expression level to 10 or lower. The patient underwent HSCT twice, and FUS-ERG expression decreased rapidly, especially after human leukocyte antigen (HLA) haploidentical HSCT. Although the patient suffered multiple early recurrences after HSCTs, this case suggests that HLA-haploidentical HSCT is one of the options for AML with $t(16 ; 21)(p 11 ; q 22)$, even though the benefits are limited. Further research is needed to optimize the course of treatment including pre-transplant treatment, HSCT, and post-transplant treatment strategies (Trial registration: jRCTs051180119). (Journal of Hematopoietic Cell Transplantation $9(2): 65-69,2020$.)

\section{Introduction}

$\mathrm{t}(16 ; 21)(\mathrm{p} 11 ; \mathrm{q} 22)$ is a rare non-random chromosomal abnormality found in approximately $1 \%$ of acute myelogenous leukemia (AML) cases. ${ }^{1}$ This chromosomal rearrangement results in FUS-ERG fusion transcripts. ERG, located at chromosome $21 \mathrm{q} 22$, is a member of the ets oncogene family. ${ }^{2}$ Correct $E R G$ gene dosage is critical to maintain hematopoietic stem cell function. FUS, at chromosome 16p11, encodes an RNA-binding protein with extensive amino-acid sequence homology to EWS, which is derived from Ewing sarcoma. ${ }^{3}$
The FUS-ERG gene is produced by fusion of the 5 , end of FUS to the 3' end of ERG. ${ }^{4}$ The FUS fusion domain regulates DNA-binding activity of the FUS-ERG chimeric protein, resulting in weaker transcriptional activation than that with normal ERG proteins. ${ }^{5}$ Dysregulation of normal ERG transcription by FUS-ERG fusion might contribute to the pathogenesis of AML with $\mathrm{t}(16 ; 21)(\mathrm{p} 11 ; \mathrm{q} 22)$.

AML with $t(16 ; 21)(\mathrm{p} 11 ; \mathrm{q} 22)$ occurs more often in younger individuals for reasons not fully understood. Buchanan et al. compiled 78 cases and reported that the median age was 25 years. ${ }^{6}$ AML with $\mathrm{t}(16 ; 21)$ (p11;q22) has

Submitted December 27, 2019; Accepted January 31, 2020; Published online, April 15, 2020. (Handling Editor: Masao Ogata, Oita University Hospital)

Key words: Acute myelogenous leukemia, FUS-ERG, hematopoietic stem cell transplantation, reverse-transcription quantitative polymerase chain reaction

Correspondence: Yumiko Inui, Department of Medical Oncology/Hematology, Kakogawa Central City Hospital, 439 Hon-machi, Kakogawa-cho, Kakogawa, Hyogo, 675-0037, Japan. E-mail: yuinui@med.kobe-u.ac.jp dx.doi.org/10.7889/hct-19-017 (C) The Japan Society for Hematopoietic Cell Transplantation. 
Table1. Laboratory data at the fierst visit

\begin{tabular}{|c|c|c|c|c|c|}
\hline \multicolumn{2}{|c|}{ Peripheral Blood } & \multicolumn{2}{|c|}{ Blood Chemistory } & \multicolumn{2}{|c|}{ Serology } \\
\hline WBC & $505,400 / \mu \mathrm{L}$ & TP & $6.7 \mathrm{~g} / \mathrm{dL}$ & CRP & $0.76 \mathrm{mg} / \mathrm{dL}$ \\
\hline Seg & $3 \%$ & ALB & $4.2 \mathrm{~g} / \mathrm{dL}$ & $\lg G$ & $797 \mathrm{mg} / \mathrm{dL}$ \\
\hline Lym & $5 \%$ & AST & $27 \mathrm{U} / \mathrm{L}$ & $\lg A$ & $83 \mathrm{mg} / \mathrm{dL}$ \\
\hline Mono & $1 \%$ & ALT & $18 \mathrm{U} / \mathrm{L}$ & IgM & $61 \mathrm{mg} / \mathrm{dL}$ \\
\hline Eo & $0 \%$ & LDH & $1,813 \mathrm{U} / \mathrm{L}$ & & \\
\hline Baso & $0 \%$ & ALP & 198 U/L & & \\
\hline Blast & $91 \%$ & T-Bil & $0.41 \mathrm{mg} / \mathrm{dL}$ & \multicolumn{2}{|c|}{ Bone Marrow } \\
\hline $\mathrm{RBC}$ & $389 \times 10^{4} / \mu \mathrm{L}$ & BUN & $11.3 \mathrm{mg} / \mathrm{dL}$ & NCC & $5 \times 10^{4} / \mu \mathrm{L}$ \\
\hline $\mathrm{Hb}$ & $11.8 \mathrm{~g} / \mathrm{dL}$ & $\mathrm{Cr}$ & $0.73 \mathrm{mg} / \mathrm{dL}$ & MgK & $9 / \mu \mathrm{L}$ \\
\hline $\mathrm{Ht}$ & $35.9 \%$ & UA & $3.1 \mathrm{mg} / \mathrm{dL}$ & $\mathrm{M} / \mathrm{E}$ retio & 0.94 \\
\hline MCV & $92 \mathrm{fl}$ & $\mathrm{Na}$ & $140 \mathrm{mmol} / \mathrm{L}$ & Myeloid & $3.2 \%$ \\
\hline $\mathrm{MCH}$ & $30.3 \mathrm{pg}$ & K & $4 \mathrm{mmol} / \mathrm{L}$ & Erythloid & $3.4 \%$ \\
\hline $\mathrm{MCHC}$ & $32.9 \%$ & $\mathrm{Cl}$ & $103 \mathrm{mmol} / \mathrm{L}$ & Eo & $0.4 \%$ \\
\hline Plt & $14.4 \times 10^{4} / \mu \mathrm{L}$ & $\mathrm{Ca}$ & $9 \mathrm{mg} / \mathrm{dL}$ & Baso & $0.2 \%$ \\
\hline PT & $75 \%$ & $\mathrm{P}$ & $3.7 \mathrm{mg} / \mathrm{dL}$ & mono & $1.6 \%$ \\
\hline APTT & $33.1 \mathrm{sec}$ & & & Ly & $10.6 \%$ \\
\hline Fib & $323 \mathrm{mg} / \mathrm{dL}$ & & & Promono & $3.8 \%$ \\
\hline FDP & $9.6 \mu \mathrm{g} / \mathrm{mL}$ & & & Leukemic blast & $76.6 \%$ \\
\hline
\end{tabular}

Figure 1. Appearance of blasts. (A) Appearance of blasts in the bone marrow (BM). (a) May-Giemsa-stained leukemic blasts with azurophilic granules and Auer bodies in the cytoplasm. (b) A total of $46 \%$ of leukemic blasts were positive for myeloperoxidase (MPO). (c) A fraction of leukemic blasts was non-specific esterase ( $a$-naphthyl butyrate)-positive. (B) Gbanded karyotype of BM cells. The karyotype was 46,XY,-16, der (21)t(16;21) (p11.2;q22). The arrow indicates rearranged chromosomes.
(A)

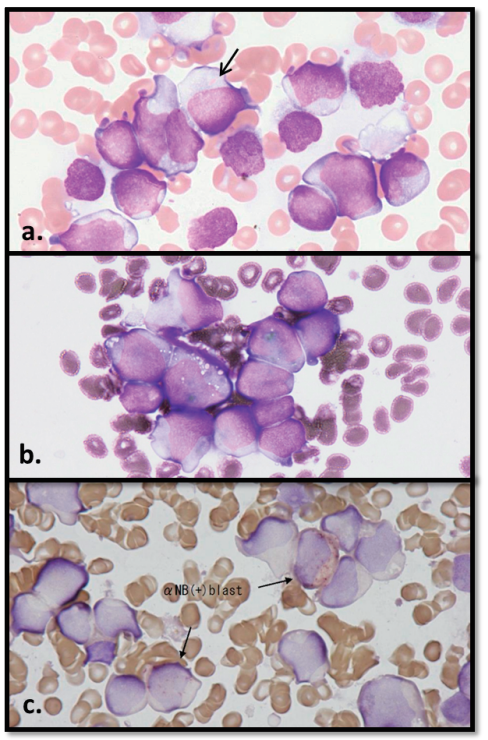

(B)

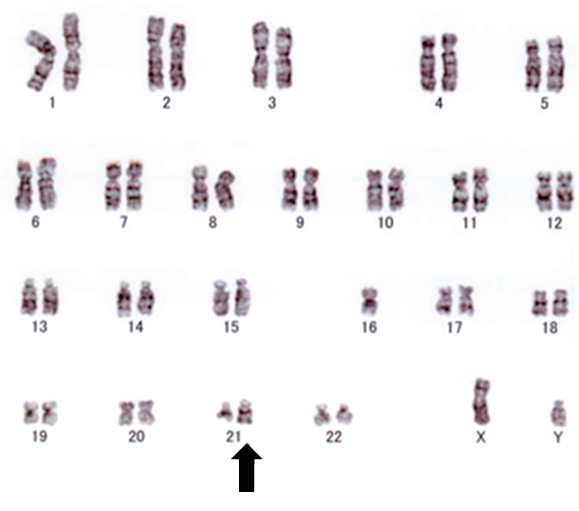

a high risk of relapse and poor prognosis. We describe a patient with AML with $\mathrm{t}(16 ; 21)$ (p11;q22) who had undergone successful monitoring of FUS-ERG fusion transcripts by real-time quantitative polymerase chain reaction (RT-qPCR) and consider the effectiveness of hematopoietic stem cell transplantation (HSCT) for AML with this chromosomal rearrangement.

\section{Case}

\section{Initial therapy}

A 32-year-old man presented to a urology department with complaints of fever and a right scrotal mass. A blood test revealed leukocytosis with lactase dehydrogenase elevation (Table 1). Bone marrow (BM) examination revealed an increase in abnormal myeloblasts with a low nucleus: cytoplasm ratio, accounting for $52 \%$ of nucleated cells (Figure 1A). The patient was diagnosed with AML (French-American-British Classification; M1) and received induction chemotherapy with cytarabine infusion for 7 days $\left(100 \mathrm{mg} / \mathrm{m}^{2} /\right.$ day $)$ and idarubicin for 3 days $\left(12 \mathrm{mg} / \mathrm{m}^{2} /\right.$ day $)$. Cytogenetic analysis revealed a 46,XY,-16, der (21) t (16;21) (p11.2;q22) [20] karyotype (Figure 1B). 


\section{Marker Patient}

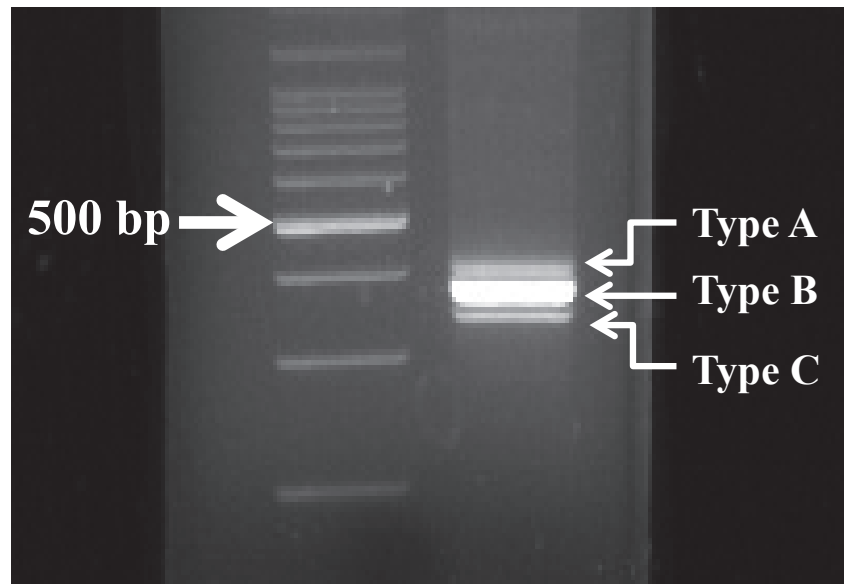

Figure 2. Electrophoresis of the amplified products. Reverse transcription polymerase chain reaction analysis of FUS-ERG transcripts. Three types of transcripts were detectable in the same specimen.

\section{RT-qPCR}

We prepared RT-qPCR primers to detect the FUS-ERG transcript to accurately evaluate minimal residual disease. The RNeasy Mini Kit (QIAGEN, Hilden, Germany) was used to extract total RNA from BM cells. From $1 \mu \mathrm{g}$ of total RNA, cDNA was synthesized by reverse transcriptase using the SuperScript VILO cDNA Synthesis Kit (Invitrogen, Carlsbad, CA, USA). The first PCR was performed using AmpliTaq Gold 360 Master Mix (Applied Biosystems, Foster City, CA, USA) and primers (4F1, CTATGGACAGCAGGACCGTG; 8R, CATAGTAGTAACGGAGGGCG) as previously describe. ${ }^{?}$

Electrophoresis of the amplified products is shown in Figure 2. In a previous study, one major band (type B; exon 7 of FUS fused in-frame to exon 9 of $E R G$ ) and two minor bands (type A; exon 7 of FUS fused out-frame to exon 9 of ERG; type C, exon 6 of FUS fused out-frame to exon 9 of $E R G$ ) were detected in most patients with AML with $\mathrm{t}(16 ; 21) .^{7}$ Our patient also showed three chimeric transcripts, specifically type B (380 bp), type A (424 bp), and type C (345 bp).

FUS-ERG expression was measured by RT-qPCR using Fast Advanced Master Mix (Applied Biosystems). Primers were designed to detect all three transcripts. The following PCR primers were used: 4F1, CTATGGACAGCAGGACCGTG (forward), and ERG, CCAGGAGGAACTGCCAAAG (reverse). The probe sequence was as follows: FAM-GTTACAACCGCAGCAGTGGTGGCTATGAACCCAGATAMRA. Glyceraldehyde 3-phosphate dehydrogenase (GAPDH; Corresponding TaqMan Assay ID: Hs99999905_ m1) was used as a control for the presence of amplifiable RNA.

\section{First HSCT}

The patient was not in complete remission $(\mathrm{CR})$ after initial induction chemotherapy and received a second course including 7 days of cytarabine infusion $\left(100 \mathrm{mg} / \mathrm{m}^{2} /\right.$ day $)$ and 5 days of daunorubicin $\left(50 \mathrm{mg} / \mathrm{m}^{2} /\right.$ day $)$. Although he was in hematological CR after this, cytogenetic CR was not achieved; the karyotype was 46,XY,-16,der (21) t (16;21) (p11.2;q22) [2]/46,XY [18].

He received an allogeneic peripheral blood stem cell transplantation (allo-PBSCT) in the first remission using a graft from an HLA-matched related donor 4 months after diagnosis. Hematopoietic stem cells $\left(\mathrm{CD} 34^{+}\right.$cells; $3.6 \times 10^{6} / \mathrm{kg}$ ) were transfused after myeloablative conditioning chemotherapy with a busulfan (BU)/cyclophosphamide (CY) regimen $(3.2 \mathrm{mg} / \mathrm{kg} \mathrm{BU}$, days -5 to $-2 ; 60 \mathrm{mg} / \mathrm{kg} \mathrm{CY}$, days -7 and -6$)$. Ciclosporin and mycophenolate mofetil (MMF) were administered as graft-versus-host disease (GVHD) prophylaxis. He achieved cytogenetic CR for the first time after the transplant.

Changes in transcript levels are shown in Figure 3. Immunosuppressive drugs for GVHD prophylaxis were tapered to obtain the desired graft-versus-leukemia effect. However, molecular CR was never achieved. Hematological relapse was detected on day 138 after PBSCT. In addition to $\mathrm{t}(16 ; 21)$, cytogenetic analysis revealed complex karyotype abnormalities at the time of relapse: 45,XY,-1, add (7) (q22), add (8) (q11.2), add (12) (q24.1),-16, der (21) ?t (16;21) (p11.2;q22), +der (?) t (?;1) (?;q21) [20].

\section{Second HSCT}

The patient underwent a second allo-PBSCT in the second remission using a graft from an HLA-haploidentical related donor. This HLA-haploidentical HSCT using post-transplant CY was performed as part of a clinical trial, approved by Kobe University Clinical Research Ethical Committee (approval number: jRCTs051180119) and conducted in accordance with the Declaration of Helsinki. The patient provided written informed consent prior to enrollment. Hematopoietic stem cells $\left(\mathrm{CD} 34^{+}\right.$cells; $\left.6.5 \times 10^{6} / \mathrm{kg}\right)$ were transfused after reduced intensity conditioning (RIC) chemotherapy with a fludarabine $(\mathrm{Flu}) / \mathrm{BU} / \mathrm{CY}$ regimen $\left(30 \mathrm{mg} / \mathrm{m}^{2} \mathrm{Flu}\right.$, day -6 to $-2 ; 14.5 \mathrm{mg} / \mathrm{kg} \mathrm{CY}$, days -6 and $-5 ; 3.2 \mathrm{mg} / \mathrm{kg} \mathrm{BU}$, days -3 and -2$)$. Post-transplant high-dose CY $(50 \mathrm{mg} / \mathrm{kg}$ CY, days 3-4), tacrolimus, and MMF were administered as 
Figure 3. Change in the relative expression of FUS-ERG. Change in the relative expression of FUS-ERG with treatment in the patient. We used $G A P D H$ as the internal standard gene to standardize the expression of FUS-ERG. Multiagent chemotherapy did not reduce the expression level to $10^{3} \times 10^{-6}$ or below. After the second hematopoietic stem cell transplantation (HSCT) with cells derived from an HLA-haploidentical donor, FUS-ERG expression decreased rapidly, despite the reduced intensity of this HSCT compared to that with the first HSCT with cells derived from the HLA-matched donor.

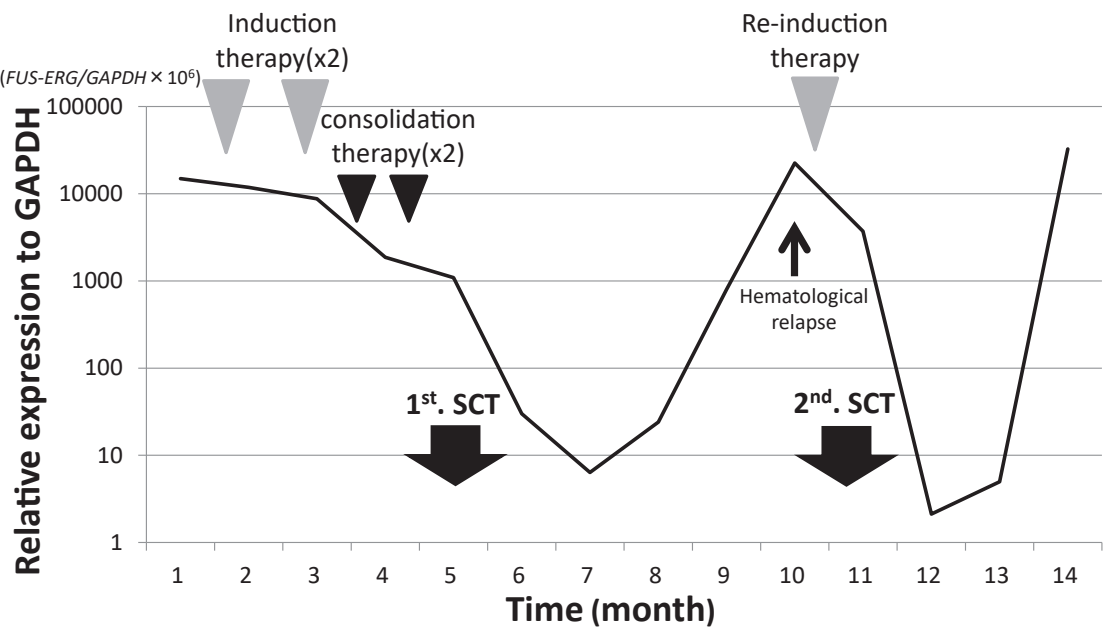

GVHD prophylaxis. After the second PBSCT, the patient achieved cytogenetic $\mathrm{CR}$ but not molecular CR; small quantities of FUS-ERG transcripts were detected by RT-qPCR. Nevertheless, treatment had a strong impact on FUS-ERG levels, which decreased more rapidly than those detected after the first HSCT with an HLA-matched related donor, despite RIC and the new complex chromosomal aberrations. The patient had mixed chimerism (91.2\% second donor cells and $8.8 \%$ first donor cells) by day 63 . The immunosuppressive drugs were tapered rapidly to eradicate leukemic cells with careful attention to GVHD. However, an explosive increase in FUS$E R G$ fusion gene levels was detected, and the patient died of primary disease 125 days after the second PBSCT.

\section{Discussion}

We describe a patient with AML with $\mathrm{t}(16 ; 21)$ (p11;q22) who underwent successful monitoring of FUS-ERG fusion transcripts by RT-qPCR. Standard multiagent chemotherapy could not reduce FUS-ERG expression, whereas HSCT decreased expression to the quantitative limit of sensitivity or lower. Unfortunately, the leukemia was refractory to treatment despite prompt initiation of the first and second rounds of HSCT. However, HLA-haploidentical HSCT remarkably and instantaneously reduced FUS-ERG expression despite the disadvantageous conditions. HSCT had limited benefits, even though it was the most effective treatment.

In most cases of haploidentical SCT using post-transplant $\mathrm{CY}$, fevers of over $38^{\circ} \mathrm{C}$ can occur from the next day after SCT and persist to approximately the fifth day. This noninfectious fever is known as "haplo-immunostorm" syndrome (HIS) because of a decline in fever following post-transplant $\mathrm{CY}^{8}$
HIS occurs only in patients receiving at least $10^{8} \mathrm{CD}^{+}$cells/ $\mathrm{kg}$, and haploidentical PBSCT tends to cause HIS in comparison to haploidentical bone marrow transplantation. ${ }^{8-9}$ Intriguingly, HIS was suggested to activate NK cells and might mediate tumor responses. ${ }^{9}$ Although the FUS-ERG transcript level decreased more rapidly after haploidentical SCT compared to that with HLA-matched SCT in our patient, we assume that HIS might be the reason.

Noort et al. performed HSCTs on 22 AML cases with $t$ $(16 ; 21)(\mathrm{p} 11 ; \mathrm{q} 22)$, and the 4-year event-free survival (EFS) was $15 \% .{ }^{10}$ Qin et al. reported that the 2-year EFS and 2-year overall survival (OS) after HSCT in 14 cases were $30.8 \%$ and $46.2 \%$, respectively. ${ }^{11}$ Although they did not analyze the 4-year EFS and OS, values of $30 \%$ and $33 \%$, respectively, could be deduced from Kaplan-Meier curves. In a study by Qin et al., all cases except three, which received HLAmatched sibling HSCT, received HLA-haploidentical HSCT. Several studies focusing on the HSCT outcome for high-risk patients with AML have shown that HLA-haploidentical HSCT and HLA-matched sibling HSCT are equivalent in efficacy or that HLA-haploidentical HSCT might have superior antileukemia effects. ${ }^{12-13}$ Although these suggested that HLAhaploidentical HSCT may be associated with a low incidence of relapse as compared to HLA-matched sibling HSCT in high-risk AML patients, currently, it is impossible to say definitely that HLA-haploidentical HSCT is superior in terms of GVHD-free-relapse-free survival. Including improvements in the outcomes of HLA-haploidentical HSCT, the selection of donors for HSCT in high-risk AML remains a critical issue.

Interestingly, all-trans retinoic acid (ATRA) may be effective against AML with $\mathrm{t}(16 ; 21)(\mathrm{p} 11 ; \mathrm{q} 22)$. Sotoca et al. discovered that FUS-ERG colocalizes to a similar region as the 
nuclear receptor RARA, and treatment of $\mathrm{t}(16 ; 21)$ (p11;q22) cells with ATRA results in cell differentiation and apoptosis, consistent with the onset of post-differentiation cell death. ${ }^{14}$ If ATRA has beneficial effects in AML with $t(16 ; 21)$ (p11;q22), it may become an attractive option for pretransplant and post-transplant treatment.

Here, we describe the potential and limitation of HLA-haploidentical HSCT for the treatment of AML with $\mathrm{t}(16 ; 21)$ (p11;q22), and utilities of monitoring FUS-ERG fusion transcripts by RT-qPCR. Due to the rarity of this AML subtype, it is difficult to conduct large clinical trials. However, further research is needed to optimize the course of treatment including pre-transplant treatment, HSCT, and post-transplant treatment.

\section{Acknowledgments}

We would like to thank Editage (www.editage.com) for English language editing.

\section{Authors' contributions}

YI, the main author, wrote and revised the manuscript and provided direct care to the patient. KM, HM, and YN performed the RT-PCR. TN and RS provided direct care to the patient and collected data. KY interpreted data and revised the manuscript. AO provided direct care to the patient and revised and gave final approval of the manuscript.

\section{Conflict of interest disclosure}

The authors declare no conflict of interest

\section{References}

1. Jekarl DW, Kim M, Lim J, et al. CD56 antigen expression and hemophagocytosis of leukemic cells in acute myeloid leukemia with $\mathrm{t}(16 ; 21)$ ( $\mathrm{p} 11 ; \mathrm{q} 22)$. International Journal of Hematology. 2010; 92: 306-313.

2. Reddy ES, Rao VN, Papas TS. The erg gene: a human gene related to the ets oncogene. Proceedings of the National Academy of Sciences of the United States of America. 1987; 84: 6131-6135.

3. Rabbitts TH, Forster A, Larson R, Nathan P. Fusion of the dominant negative transcription regulator $\mathrm{CHOP}$ with a novel gene FUS by translocation $t(12 ; 16)$ in malignant liposarcoma. Nature Genetics. 1993; 4: 175-180.

4. Ichikawa H, Shimizu K, Hayashi Y, Ohki M. An RNA-binding protein gene, TLS/FUS, is fused to ERG in human myeloid leukemia with $\mathrm{t}(16 ; 21)$ chromosomal translocation. Cancer Research. 1994; 54: 2865-2868.

5. Prasad DD, Ouchida M, Lee L, Rao VN, Reddy ES. TLS/FUS fusion domain of TLS/FUS-erg chimeric protein resulting from the $t(16 ; 21)$ chromosomal translocation in human myeloid leukemia functions as a transcriptional activation domain. Oncogene. 1994; 9: 3717-3729.

6. Buchanan J, Tirado CA. A t $(16 ; 21)(\mathrm{p} 11 ; \mathrm{q} 22)$ in acute myeloid leukemia (AML) resulting in fusion of the FUS/TLS and ERG genes: A review of the literature. Journal of the Association of Genetic Technologists. 2016; 42: 24-33.

7. Kong XT, Ida K, Ichikawa $\mathrm{H}$, et al. Consistent detection of TLS/FUS-ERG chimeric transcripts in acute myeloid leukemia with $\mathrm{t}(16 ; 21)(\mathrm{p} 11 ; \mathrm{q} 22)$ and identification of a novel transcript. Blood. 1997; 90: 1192-1199.

8. Sugita J, Kawashima N, Fujisaki T, et al. Transplantation, HLA-haploidentical peripheral blood stem cell transplantation with post-transplant cyclophosphamide after busulfan-containing reduced-intensity conditioning. Biology of Blood and Marrow Transplantation: Journal of the American Society for Blood and Marrow Transplantation. 2015; 21: 1646-1652.

9. Colvin GA, Berz D, Ramanathan M, et al. Nonengraftment haploidentical cellular immunotherapy for refractory malignancies: tumor responses without chimerism. Biology of Blood and Marrow Transplantation: Journal of the American Society for Blood and Marrow Transplantation. 2009; 15: 421-431.

10. Noort S, Zimmermann M, Reinhardt D, et al. Prognostic impact of $\mathrm{t}(16 ; 21)(\mathrm{p} 11 ; \mathrm{q} 22)$ and $\mathrm{t}(16 ; 21)(\mathrm{q} 24 ; \mathrm{q} 22)$ in pediatric AML: a retrospective study by the I-BFM Study Group. Blood. 2018; 132: 1584-1592.

11. Qin YZ, Chen Y, Xu LP, et al. Outcome and minimal residual disease monitoring in patients with $t(16 ; 21)$ acute myelogenous leukemia undergoing allogeneic hematopoietic stem cell transplantation. Biology of Blood and Marrow Transplantation: Journal of the American Society for Blood and Marrow Transplantation. 2018; 24: 163-168.

12. Salvatore D, Labopin M, Ruggeri A, et al. Outcomes of hematopoietic stem cell transplantation from unmanipulated haploidentical versus matched sibling donor in patients with acute myeloid leukemia in first complete remission with intermediate or high-risk cytogenetics: a study from the Acute Leukemia Working Party of the European Society for Blood and Marrow Transplantation. Haematologica. 2018; 103: 1317-1328.

13. Chang YJ, Wang Y, Liu YR, et al. Haploidentical allograft is superior to matched sibling donor allograft in eradicating pretransplantation minimal residual disease of AML patients as determined by multiparameter flow cytometry: a retrospective and prospective analysis. Journal of Hematology \& Oncology. 2017; 10: 134.

14. Sotoca AM, Prange KH, Reijnders B, et al. The oncofusion protein FUS-ERG targets key hematopoietic regulators and modulates the all-trans retinoic acid signaling pathway in t (16;21)acute myeloid leukemia. Oncogene. 2016; 35: 1965-1976. 